\title{
A LOGÍSTICA COMO INSTRUMENTO DE APOIO AO DESENVOLVIMENTO REGIONAL - O CASO DO ASSENTAMENTO SÃO BENTO III EM MIRANTE DO PARANAPANEMA (S.P)
}

\section{Carlos Lourenço Atanázio Trombini ${ }^{1}$}

\author{
Edilene Mayumi Murashita Takenaka ${ }^{2}$
}

\begin{abstract}
RESUMO
Este trabalho de pesquisa foca abordar de forma direta a questão do desenvolvimento regional, e como a logística pode ser uma importante ferramenta de apoio a esse processo, tendo como foco a produção familiar no município de Mirante do Paranapanema, localizado na região do Pontal do Paranapanema a $558 \mathrm{~km}$ de São Paulo, o tema é de importante relevância para o contexto agrícola nacional, uma vez que a relação produção agrícola $x$ desenvolvimento logístico tem sido debatida cada vez mais. Para o desenvolvimento do trabalho, as metodologias utilizadas serão as de pesquisa bibliográfica, pesquisa de campo, analise qualitativa e quantitativa de dados. A pesquisa bibliográfica terá como fontes livros (clássicos e contemporâneos), artigos, sites, todos relativos a reflexão cientifica voltada para a temática da produção familiar. Será feita a pesquisa de campo com os pecuaristas familiares de Mirante do Paranapanema, objetivando visualizar a questão da infraestrutura logística e de que forma esta contribui com desenvolvimento do objeto de pesquisa dentro das particularidades de cada ambiente, entendida essa questão, será utilizada a literatura para demonstrar como a logística poderia contribuir como instrumento de desenvolvimento econômico, social e ambiental.
\end{abstract}

PALAVRAS-CHAVE: Desenvolvimento regional, logística, assentamentos e produção familiar.

\section{LOGISTICS SUPPORT AS A TOOL TO REGIONAL DEVELOPMENT - THE FIXING CASE SAINT BENEDICT III IN THE MIRANTE DO PARANAPANEMA (S.P)}

\begin{abstract}
\footnotetext{
${ }^{1}$ Bacharel em Administração, UNOESTE discente. carlos.trombini@bol.com.br.

2 Doutora em Economia, UNOESTE docente. edilene@unoeste.br.
}

This research focuses on addressing directly the issue of regional development and how logistics can be an important support to the process tool, focusing on the family farms in the municipality of Mirante do Paranapanema, located in the Pontal do Paranapanema region to $558 \mathrm{~km}$ from São Paulo, the topic is important relevance to the national agricultural context, since the $x$ agricultural logistics 


\section{Periódica Eletranica \\ Fórum Ambiental}

da Alta Paulista
Volume 11, Número 02, 2015

Campo, Agronegócio

e as Práticas Sustentáveis

development production relationship has increasingly been debated. For the development work, the methodologies used are the bibliographic research, field research, qualitative and quantitative data analysis. The literature will have sources like books (classical and contemporary), articles, websites, all related to scientific reflection focused on the theme of family farming. Field research with the family farmers of Mirante do Paranapanema will be aiming view the issue of logistics infrastructure and how this contributes to research object development within the particularities of each environment, understood this question, the literature will be used to demonstrate how the logistics could contribute to economic, social and environmental development tool.

KEYWORDS: Regional development, logistics, settlement and family production.

\title{
APOYO LOGÍSTICO COMO HERRAMIENTA PARA EL DESARROLLO REGIONAL - EL CASO DE FIJACIÓN SAN BENITO III EN EL MIRANTE DO PARANAPANEMA (S.P)
}

\begin{abstract}
RESUMEN
Esta investigación se centra en abordar directamente el tema del desarrollo regional y cómo logística puede ser un apoyo importante a la herramienta de proceso, centrándose en las granjas familiares en el municipio de Mirante do Paranapanema, situada en el Pontal do Paranapanema región a $558 \mathrm{~km}$ de São Paulo, el tema es importante relevancia en el contexto agrícola nacional, ya que la logística $x$ agrícolas relación de producción de desarrollo cada vez más se ha debatido. Para el trabajo de desarrollo, las metodologías utilizadas son la investigación bibliográfica, investigación de campo, análisis de datos cualitativos y cuantitativos. La literatura tendrá fuentes como libros (clásicas y contemporáneas), artículos, páginas web, todos ellos relacionados con la reflexión científica se centró en el tema de la agricultura familiar. La investigación de campo con los agricultores familiares de Mirante do Paranapanema se pretende ver el tema de la infraestructura logística y cómo esto contribuye al desarrollo objeto de investigación dentro de las particularidades de cada entorno, entendida esta pregunta, la literatura se utilizará para demuestran cómo la logística podrían contribuir a la herramienta de desarrollo económico, social y ambiental.
\end{abstract}

PALABRAS CLAVE: Desarrollo Regional, de logística, de liquidación y de producción familiar.

\section{INTRODUÇÃO}

A agricultura representa hoje no Brasil um processo fundamental de desenvolvimento, no entanto é necessário melhoria do processo logístico que a atende, uma vez que este é uma ferramenta útil para a busca da sustentabilidade.

A logística é uma das ferramentas das ciências sociais aplicadas que pode auxiliar no desenvolvimento de diversas esferas da sociedade, uma vez que contribui com a eficiência dos fluxos de produtos e informações, agregando valor ao processo e consequentemente proporcionando maior competitividade aos setores que a utilizam de maneira estruturada. 
Segundo Junqueira (1999), o sistema logístico estabelece a integração dos fluxos físicos e de informações, responsáveis pelas movimentações de materiais e produtos. Assim a cadeia logística é estruturada para atender uma determinada organização, associação e mercado, melhorando as relações entre os membros da cadeia.

No contexto da agricultura familiar a logística poderia ser implantada como instrumento de apoio integrando o pequeno produtor e pecuarista familiar aos seus principais fornecedores, centros de armazenagem e clientes, desde que a mesma tenha sua cadeia estruturada. Seria esta uma área da gestão que poderia agregar valor aos produtos agrícolas e reduzir custos dos produtores.

No município de Mirante do Paranapanema (S.P), hoje com uma população de 17.059 pessoas encontra-se o objeto de pesquisa, o assentamento São Bento III, este conta com 44 lotes que variam de 15 a 20 hectares cada, sendo um dos 35 assentamentos estabelecidos em Mirantes. É importante observar que o município esta na região do Pontal do Paranapanema, conhecida pela grande quantidade de conflitos relativos à reforma agraria, além de ser reconhecidamente uma das regiões mais pobres do Estado de São Paulo. (LIMA, 2015).

A região do Pontal do Paranapanema é caracterizada por possuir grande número de terras improdutivas, devolutas e griladas ${ }^{3}$ por latifúndios, dos 88 assentamentos estabelecidos na região, o município de Mirante do Paranapanema é o que mais concentra assentamentos, 33\% do total, (PIMENTEL, 2014). Ainda é importante ressaltar que Mirante do Paranapanema tem 1.242 famílias assentadas, 40 delas no Assentamento São Bento III.

O processo de desenvolvimento rural brasileiro demanda a utilização de recursos e ferramentas que até então tem sido utilizadas de forma comercial apenas pelos grandes latifúndios e empresas focadas no agronegócio e pecuária de larga escala comercial. É preciso, para que os pequenos proprietários rurais tenham condições de se desenvolver, disponibilizar de maneira estruturada mecanismos que Ihes permitam crescer comercialmente, é necessário verificar se a logística adaptada

\footnotetext{
${ }^{3}$ O INCRA (Instituto Nacional de Colonização e reforma Agrária) considera improdutiva a propriedade que não atinge grau de eficiência de exploração ou grau de utilização da terra. Grilagem, nada mais é do que a apropriação de terras públicas por meio da falsificação de documentos. Terras devolutas são terras públicas que em momento algum pertenceram a um particular.
} 
a realidade dos produtores do Assentamento São Bento III fomentaria um modelo de desenvolvimento que atenderia suas necessidades.

Analisando a realidade dos pequenos produtores do assentamento São Bento III, e tendo como base o referencial teórico voltado para os conceitos como de logística de suprimentos, logística de produção, logística de distribuição e logística reversa, Matriz SWOT, crescimento e desenvolvimento. A pesquisa busca entender de que forma a logística, como ferramenta, pode contribuir de maneira positiva com os produtores familiares do assentamento São Bento III, fomentando o crescimento econômico, e corroborando com o desenvolvimento regional.

Dentre os setores da economia brasileira que mais contribuem para o PIB nacional, o agronegócio sem duvida é o que mais gera riquezas ao Brasil, a agricultura e pecuária de larga escala contribuem para a balança comercial brasileira, a Estada por sua vez incentiva o setor promovendo politicas públicas que fortaleçam ainda mais o segmento. Contrariamente, não observamos que o governo se dedique de maneira proporcional aos pequenos proprietários rurais, não se destina a mesma atenção no planejamento e execução de politicas públicas, apesar da importância que estes têm na sociedade, na economia, no abastecimento de alimentos e até em um sistema produtivo mais responsável ambientalmente.

[...] a agricultura familiar destaca-se como uma forma de vida de milhares de
homens e mulheres que resiste ao longo do tempo, ao processo excludente
de politicas governamentais, e que buscam, dentro de um mundo
capitalista, manter seu espaço em um mundo cada vez mais competitivo,
concorrendo em nível desigual com os empreendimentos que atual na
lógica do agronegócio. (Silva e Jesus, p.1, 2010).

A atenção por parte do Estado para as politicas publicas voltadas aos pequenos produtores rurais não tem sido eficientes, a pesquisa poderá demonstrar que mediante a atenção do governo para a estruturação da logística do São Bento III, os assentados terão mais condições de se estabelecer no mercado e tem mais capacidade competitiva.

O processo de desenvolvimento brasileiro passa obrigatoriamente pela agricultura, até mesmo pelas próprias características do país, extensão territorial, clima, mão de obra farta e barata, além de incentivos governamentais. A escolha pela produção agrícola para ser a grande atividade impulsionadora da economia brasileira pode ser comprovada historicamente. Verdadeiramente o Brasil, desde a 
chegada dos colonizadores portugueses tem sido utilizado como um grande campo de exploração de recursos naturais.

A agricultura familiar também exerce papel importante no crescimento econômico brasileiro, além de contribuir significativamente com o PIB (Produto Interno Bruto) nacional é engrenagem fundamental na produção de alimentos para o Brasil.

A agricultura familiar é hoje responsável por $70 \%$ dos alimentos consumidos pelos brasileiros. De acordo com o Censu Agropecuário de 2006 - o mais recente feito no país -, são fornecidos pela agricultura familiar os principais alimentos consumidos pela população brasileira: $87 \%$ da produção nacional de mandioca, $70 \%$ da produção de feijão, $46 \%$ do milho, $38 \%$ do café, $34 \%$ do arroz, $58 \%$ do leite, possuíam $59 \%$ do plantel de suínos, $50 \%$ do plantel de aves, $30 \%$ dos bovinos, e produzem $21 \%$ do trigo. No Censu Agropecuário de 2006 foram identificados 4,3 milhões de estabelecimentos de agricultores familiares, o que representa $84,4 \%$ dos estabelecimentos agropecuários brasileiros. Este segmento produtivo responde por $10 \%$ do Produto Interno Bruto (PIB), 38\% do Valor Bruto da Produção Agropecuária e $74,4 \%$ da ocupação de pessoal no meio rural. (Ministério do Desenvolvimento Agrário, 2011, s/p)

Lesbaupin (2012), afirma que $70 \%$ dos alimentos que são consumidos advêm da produção agrícola familiar, essa constatação demonstra que tal agricultura demanda atenção superior a dada atualmente. A figura $n$ 1, (pag. 6) compara características da produção familiar de alimentos com a produção do agronegócio.

Figura 1 - Comparação da produção das características da produção familiar de alimentos com a produção do agronegócio.

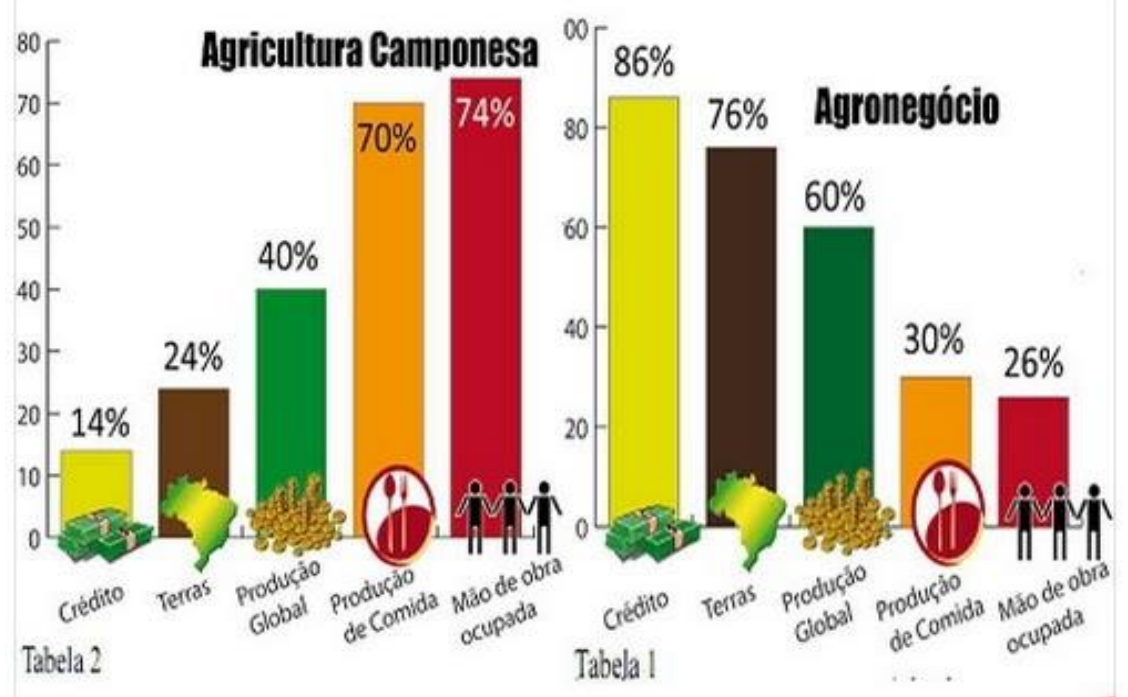

Fonte: Bertoni (2014) 
Observa-se a real importância que a agricultura familiar tem enquanto instrumento de contribuição para o crescimento econômico do país e também para a própria estrutura de sobrevivência nacional, seja na produção de alimentos e oportunidades de trabalho por exemplo. O referencial teórico também demonstra que apesar da importância da agricultura familiar, os recursos têm sido destinados de maneira desproporcional ao modelo de produção capitalista de agronegócio e pecuária em grande escala.

Na safra de 2003-2004, o PRONAF recebeu 4,4 bilhões de reais e 0 agronegócio 33 bilhões. Na safra de 2004-2005, o PRONAF recebeu 7 bilhões de reais e o agronegócio, 38 bilhões. Para a safra de 2005-2006, foram disponibilizados 9 bilhões de reais para o PRONAF e 44 bilhões para o agronegócio (Hespanhol, 2009, p 40).

Sabe-se que a o planejamento e a efetivação do processo de infraestrutura logística contribui de sobre maneira para o crescimento econômico, social, ambiental, ou seja, para o desenvolvimento de uma organização, na realidade dos pequenos produtores rurais não é diferente, já que aproxima fornecedores de produtores, produtores de clientes e consequentemente gera a oportunidade de serem mais competitivos e terem mais recursos.

O processor logístico na agricultura representa um componente importante para o desenvolvimento do país, uma vez que o conhecimento se apresenta como ferramenta útil para a sustentabilidade (...) (S. Cabral, Menelau A. S, T. Vital, 2012, p. 1)

Evidente que investir em infraestrutura logística é importante, porém por si só não garante desenvolvimento de uma região, comunidade ou sociedade, é preciso, em paralelo, que ocorra o apoio do poder público aos pequenos produtores rurais, principalmente em relação à gestão, demonstrando aos mesmos de que maneira utilizar a logística de maneira eficaz, o apoio técnico e as ferramentas de gestão corroboram para o desenvolvimento da comunidade.

BALLOU (2010), afirma que a Matriz SWOT Strenghts (Forças), Weaknesses (Fraquezas), Opportunities (Oportunidades) e Threats (Ameaças), esta entre as técnicas mais interessantes para analisar potencialidades, aspectos críticos, qualidades e dificuldades de um processo. A Matriz SWOT será utilizada durante a pesquisa para evidenciar quais fatores devem ser melhorados ou potencializados 
para que a proposta logística seja a mais adequada à realidade do assentamento São Bento III.

Deve-se ressaltar que o apoio das ferramentas de gestão, como a Matriz SWOT contribui com o processo de analise critica ao contexto logístico que afeta o Assentamento São Bento III, e a mesma revelará de forma clara quais problemas afetam a cadeia logística, assim como indicará meios para a formação de um plano que atenda as necessidades da comunidade.

\section{OBJETIVOS}

\subsection{OBJETIVO GERAL}

Identificar se a logística poderia ser utilizada como um instrumento de apoio ao desenvolvimento regional, bem como sua contribuição para a agricultura familiar no Pontal do Paranapanema: Enfoque na produção familiar do assentamento São Bento III.

\subsection{OBJETIVOS ESPECÍFICOS}

2.2.1 Conceituar os principais aspectos ligados ao desenvolvimento regional sustentável envolvendo a responsabilidade social, logística e logística reversa;

2.2.2 Apresentar a importância da estruturação da cadeia logística para o desenvolvimento do assentamento São Bento III, analisando os grupos de pecuaristas familiares pesquisados, a partir de suas atividades produtivas;

2.2.3 Verificar como a efetivação da cadeia de logística poderia contribuir com a produção familiar, mediante a realidade encontrada entre os grupos de pecuaristas familiares pesquisados;

2.2.4 Apontar por meio da utilização da Matriz SWOT, problemas e potencialidades da cadeia de logística que interferem ou podem interferir no desenvolvimento entre os grupos de pecuaristas familiares pesquisados;

2.2.5 Traçar um plano logístico que contemple a realidade do pequeno produtor rural, o poder público e demais membros da cadeia, favorecendo o planejamento da 
pequena propriedade rural e a preservação ambiental, observando a realidade dos produtores familiares pesquisados.

\section{METODOLOGIA / MÉTODOS DE ANALISE}

Para a realização da pesquisa os métodos mais apropriados e que serão utilizados são:

- Método de Revisão Bibliográfica;

- Método de Pesquisa de Campo.

Para a busca de informações serão utilizados livros, artigos, jornais e sites de caráter cientifico voltados ao estudo da agricultura familiar, a logística, gestão e sua aplicabilidade como ferramenta para o desenvolvimento do pequeno produtor rural.

A pesquisa de campo deverá necessariamente atender aos aspectos qualitativos já que visará compreender de maneira minimalista os aspectos que compõem o fenômeno no ambiente de estudo.

Para garantir a eficácia dos estudos será preciso a sensibilidade no confronto da bibliografia estudada com a realidade observada em campo

A pesquisa também necessitará considerar os aspectos quantitativodescritivos, uma vez que serão investigados aspectos visualizados na pesquisa empírica, fatos e fenômenos, os dados coletados serão estratificados, objetivando levantamento qualitativo para analise da hipótese.

\section{RESULTADOS / HIPÓTESE}

A falta de estrutura logística tem se mostrado um gargalo para 0 desenvolvimento brasileiro, sabe-se dos diferentes problemas estruturais dessa área, essa defasagem tem inibido o crescimento nacional em diferentes esferas produtivas, (industrial, comercial e agrícola, por exemplo). É importante verificar se esta problemática tem afetado o assentamento São Bento III, se realmente esse problema for constatado a pesquisa irá propor pontos a serem melhorados do ponto de vista logístico, desde melhor organização individual dos produtores familiares, 


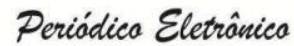

associativismo entre eles, até um apoio maior do Estado no fortalecimento de práticas que atendam as necessidades do São Bento III, podendo este servir de modelo a outros assentamentos na região do Pontal do Paranapanema.

\section{REFERÊNCIAS BIBLIOGRÁFICAS}

ALVARENGA, A. C.; NOVAES, A. G. N., Logística Aplicada. 3. ed. São Paulo: Edgar Blücher Ltda. 2000

ASSOCIAC̄̃O NACIONAL DOS DISTRIBUIDORES DE DEFENSIVOS AGRÍCOLAS E VETERINÁRIOS, Destinação Final de Embalagens Vazias de Agrotóxicos. Disponível em http://www.andav.com.br/repositorio/36.pdf, acessado em 10/11/2014

BALLOU. R. H. Logística Empresarial. 2010. São Paulo: Atlas. 388 p.

BERTONI, S. Agricultura Camponesa e Agronegócio: um comparativo gráfico esclarecedor . Disponivel em http://blogoosfero.cc/sergiobertoni/blog-do-bertoni/agricultura-camponesa-eagronegocio-um-comparativo-grafico-esclarecedor, acessado em 10/11/2014

BRASIL. Ministério do Desenvolvimento Agrário. A Agricultura Familiar no Brasil. Brasilia, 2011. Disponível em: <http://www.mda.gov.br/sistemda/noticias/com-presidenta-dilma-mda-lan\%C3\%A7arede-brasil-rural-e-entrega-114-m\%C3\%A1quinas-do-pac2-em-porto>.Acessado em 18 de mai. 2015.

CABRAL, S.; MENELAU. A.; VITAL, T. Logística de Distribuição da Produção dos Assentamentos Timbó e Granja Jumbo em Moreno, Estado de Pernambuco, São Paulo, Informações Econômicas, v. 43, p.67, mar./abr. 2013.

CARSON, R. Primavera Silenciosa. São Paulo: Gaia, 1964.

CAVALCANTI, C. Sustentabilidade da economia: Paradigmas Alternativos de Realização Economica. São Paulo: Cortez, 2003. P. 176.

COMETTI, J. L. S. Logística Reversa das Embalagens de Agrotóxicos no Brasil: Um Caminho Sustentável. 2005.152f. Dissertação (Centro de desenvolvimento Sustentável) CDS/UNB, Brasília, Distrito Federal. 2009.

FLORIANO, E. P. Planejamento Ambiental. Caderno Didático, n 6, 1ํㅡㄹ ed. Santa Rosa: 2004.

FUSSI, L. P.Metodologia Científica, Disponível em <http://profludfuzzimetodologia.blogspot.com.br/2010/03/o-que-e-pesquisa-de-campo.html> . Acesso em 10 jan 2013, 15:35

HESPANHOL, A. N. Políticas Públicas, Modernização e Crise na Agricultura Brasileira, 1999, $49 f$.

JUNQUEIRA, A. H. Tendências e Desafios da Distribuição de Produtos Hortícolas no Brasil.

Revistas de Preços Agrícolas, São Paulo, p. 5-11, maio 1999.

LAKATOS, E. M., Fundamentos da Metodologia Científica. 5 ed, São Paulo: Atlas, 2003.

LESBAUPIN, I. Por Novas Concepções de Desenvolvimento. Por Um Outro Desenvolvimento. São Paulo. 105 p. 2012.

LIMA, A. M. Agricultura Familiar no Assentamento São Bento III - Mirante do Paranapanema S.P: Uma Discussão Sobre a Importância do Planejamento Ambiental Como Ferramenta de Gestão. 2015, 176f. Dissertação (Mestrado em Meio Ambiente e Desenvolvimento Regional) UNOESTE, Presidente Prudente, São Paulo, 2015. 
PERES, R. B.; MENDIONDO, E. M. Desenvolvimento de Cenários de Recuperação como Instrumento ao Planejamento Ambiental e Urbano - Bases conceituais e Experiências Práticas, SEMINÁRIO NEUR/CEAM,Brasilia, p. 15, 2004

PERRUPATO, M. Plano Nacional de Logística de Transportes. Disponível em $<$ www.transportes.gov.br/conteudo/36991>. Acesso em 10 jan 2013, 16:59

PIMENTEL, A. E. B. Assentamentos de Reforma Agrária na Região do Pontal do Paranapanema e seus Impactos Econômicos e Sociais. 2004, 267f. Tese (Engenharia da Produção) UFSCAR, São Carlos, São Paulo, 2004.

SANTOS, A. B.D. Desenvolvimento Regional e Capital Social: Uma Abordagem para a Microrregião de Presidente Prudente - S.P. 2005. 233f. Dissertação (Mestrado em Desenvolvimento Regional e Planejamento Ambiental) FCT/UNESP, Presidente Prudente, São Paulo. 2005.

SANTOS, E. S. D. O Associativismo Como Estratégia de Gestão da Agricultura Familiar: As Associações dos Proprietários Rurais do Município de Rancharia - S.P. 2013, 159f. Dissertação (Mestrado em Meio Ambiente e Desenvolvimento Regional) UNOESTE, Presidente Prudente, São Paulo, 2013.

SILVA, J. R; JESUS, P. Os Desafios do Novo Rural e as Perspectivas da Agricultura Familiar no Brasil. 2010. Disponível em: http://connepi.ifal.edu.br/ocs/index.php/connepi/ CONNEPI2010/paper/viewFile/1407/457>. Acesso em: 30 abr. 2015.

TEIXEIRA, E. C. O Papel das Políticas Públicas no Desenvolvimento Local e na Transformação da Realidade. 2002. <http://www.dhnet.org.br/dados/cursos/aatr2/a_pdf/03_aatr_pp_papel.pdf>. Acesso em 10 jan 2013 as 13:40

VEIGA, J. E. D. Desenvolvimento Sustentável, o desafio do século XXI, prefacio de Ignacy Sachs. Rio de Janeiro, 2010. p. 226 